\title{
Appending global to local features for skin lesion classification on dermoscpic images
}

\author{
Dr. Ahlam Fadhil Mahmood* and Hamed Abdulaziz Mahmood \\ Department of Computer Engineering, College of Engineering, University of Mosul, Mosul, Iraq. \\ * Corresponding Author: ahlam.mahmood@gmail.com
}

$\begin{array}{ll}\text { Submitted } & : 12 / 05 / 2020 \\ \text { Revised } & : 30 / 06 / 2021 \\ \text { Accepted } & : 13 / 07 / 2021\end{array}$

\begin{abstract}
Skin cancer is considered the deadliest disease compared with all other kinds of cancer. In this paper, various pre- and posttreatments are proposed for improving automated melanoma diagnosis of dermoscopy images. At first, preprocessing has done to exclude unwanted parts, and a new triple-A segmentation is proposed to extract lesions according to their histogram patterns. Lastly, we suggest appending process with testing many factors for superior detection decision. This paper offers a novel approach with testing different detection rules: first, a system used fuzzy rules based on a different features, and a second test has been done by modeled local colors with bag-of-features classifier. Then, we proposed adding lesion shape on two previous systems as their global form in the first one while distributing it and appending with local color patches in the second system. For each case, different features, various color models, and many other parameters are examined to decide which settings are more discriminating. We evaluated the performance of each method carried out on ISIC2019 Challenge dermoscopic database. The novel processes with their specific parameters raised the classification accuracy to $98.26 \%$.
\end{abstract}

Keywords: Skin cancer; Dermoscopy; Fuzzy rule; Bag-of-Features; Colour feature.

\section{INTRODUCTION}

Melanoma skin cancer must be diagnosed at an early stage. Early finding and treating it can reduce the mortality and morbidity of patients (Cardoso, 2019). Digital Dermoscopy is widely considered as one of the most cost-effective methods to identify and classify skin-cancer with amplifying the lesion (Lone and Kaur, 2020), which allows detection of several surfaces and subsurface structures, which are not noticeable to the bare eye, which was used to test a skin lesion by one of the medical diagnostic strategies, like the ABCD formula (Asymmetry, uneven Border, Colour variegation and Diameter) (Tan, 2019), Menzies, pattern analysis (Masood, 2016), and the 7-point checklist methods (Sheha, 2016). All algorithms share a common four stages: (1) Artifact removal, (2) proper lesion segmentation, (3) feature extraction and selection, and (4) lesion recognition.

Two sets of dermoscopic criteria can be used to perform classification. The first one used the global features, which shown as a group of patterns (reticular, globular, cobblestone, parallel, etc.) that were founded in different pigmented skin lesions (Barata et al., 2014), whereas the second bases its decision on the local features (dots, streaks, pigment network, vascular pattern, pigmentation related structures, and globules, etc.) as shown in Figure 1. Some dermatologists perform skin lesion analysis utilizing global features only, while others test it on a local one. The main 
goal of this paper is to evaluate an accurate diagnosis system by testing different strategies. The diagnosis approaches evolve in four sequential steps. First of all, the artifacts must be removed from skin lesions. Secondly, the lesion that was segmented automatically depends on its histogram distributions according to the proposed triple-A model. Then, the $\mathrm{ABCD}$ rule (texture and color features) is extracted for a binary classification as melanoma or benign using the fuzzy classifier. A local bag of features is also tested to choose the best one, finally adding an asymmetric feature to both systems for accurate decision.

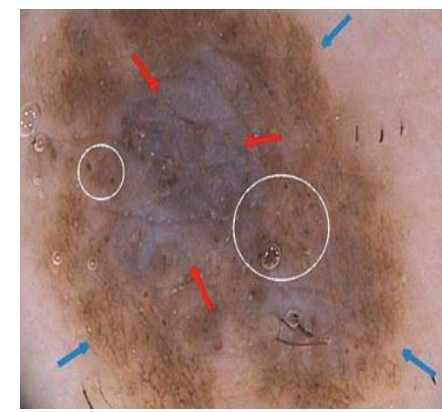

Figure 1. Dermoscopic Melanoma Features: Pigment Network, Blue-whitish veil, Dots and Globules (Barata et al., 2014)

The rest of the paper is organized as follows. Section 2 exhibits an overview of related work published in the literatures. Sections 3 and 4 describe the overall preprocessing techniques. The results acquired and their discussions are carried out in Section 5. Section 6 provides a summary and conclusion.

\section{LITERATURE REVIEW}

In the last decade, many procedures to detect melanoma have been presented. Some of these attempts were to imitate dermatologists performance by extracting and detecting most dermoscopic structures, like pigment network (Masood, 2016; Barata et al., 2014; Xie et al., 2017; Garbe et al., 2016), irregular streaks (Machado et al., 2016; Adjed, 2017), granularities, blotches (Barata et al., 2013), regression structures (Kumar and Kumanan, 2018), and blue-white veil (Sreena and Lijiya, 2018; Madooei et al., 2018). Then, these shapes can be used to score a lesion similar to dermatologists who adopted the pathway. A computerized process that demonstrates the outcomes of the 7-point checklist is founded in Ref (Kawahara et al., 2018).

However, many melanoma discovery strategies presented in writing follow a pattern identification approach (Sao et al., 2018; Sagar, 2016). Many works used global methods to classify lesions of the skin, while others utilize local one. All diagnosis systems comprise four steps, that is, artifact removal, injury segmentation, features mining, and lesion classification.

Most studies used shape features (compactness, aspect ratio with diameter) to represent injury border, color features (mean and standard deviation) in different color spaces, and texture features (gray-level co-occurrence matrix) (Kumar and Kumanan, 2018; Sagar, 2016). Authors with separate sets of characteristics and classifiers achieved various outcomes. C. Sagar system gives an accuracy of $91.8 \%$ by extract color and texture features based on neural network classifier. The overall sensitivity of his system is $95.3 \%$ for a training/testing ratio of $60 / 40$ (Sagar,2016). Xie et al. add border irregularities on complete and incomplete lesions with texture and color features. 
Their system combined fuzzy with backpropagation (BP) neural networks to improve the performance (Xie et al., 2017). Their experiments result was carried out on two diverse dermoscopy databases, that is, xanthous and caucasian races images. Barata et al. compared two approaches for revealing melanoma, one using a set of global features, and the second using a bag of features $(\mathrm{BoF})$ represented by a vector of local characteristics. As stated, their local characteristics with $\mathrm{BoF}$ accomplish slightly better outcomes as they mentioned, and the color characteristics achieve respectable results compared with texture characteristics. They carried out $\mathrm{Sp} .=75 \%$ and a $\mathrm{Se} .=100 \%$ with 176 dermoscopy images data set (Barata et al., 2014). In Ref Barata et al. (2015), the authors demonstrate a survey on four classes of features, handcrafted, dictionary-based, deep learning, and clinically inspired, to provide features guidelines for the researcher. Thompson and Jeyakumar (2018) extract BoF model using scale-invariant speeded-up robust features technique. Then, they included features on the codebook utilizing multi-SVM classifier on $1 * \mathrm{a}^{*} \mathrm{~b}$ color space. Their system provides $95.075 \%$ accuracy, $95.5 \%$ specificity, and $94.07 \%$ sensitivity by testing 305 data elements. Baji thesis (Baji, 2018) suggested melanoma detection based on three phases: ABCD rule, statistical texture analysis, and lesion symptoms. His Matlab R2016A programs verified that the contrast and entropy are powerful measurements in the characterization of the chaotic and variance of the cancerous skin images. The accuracy of the development system is $90 \%$, testing a set of 50 skin lesion images.

\section{MATERIALS AND METHODS}

The dermoscopy images classification must be done under particular conditions. Several strategies have been proposed to deal with this type of problems. The proposed system was illustrated in the block diagram of Figure 2.

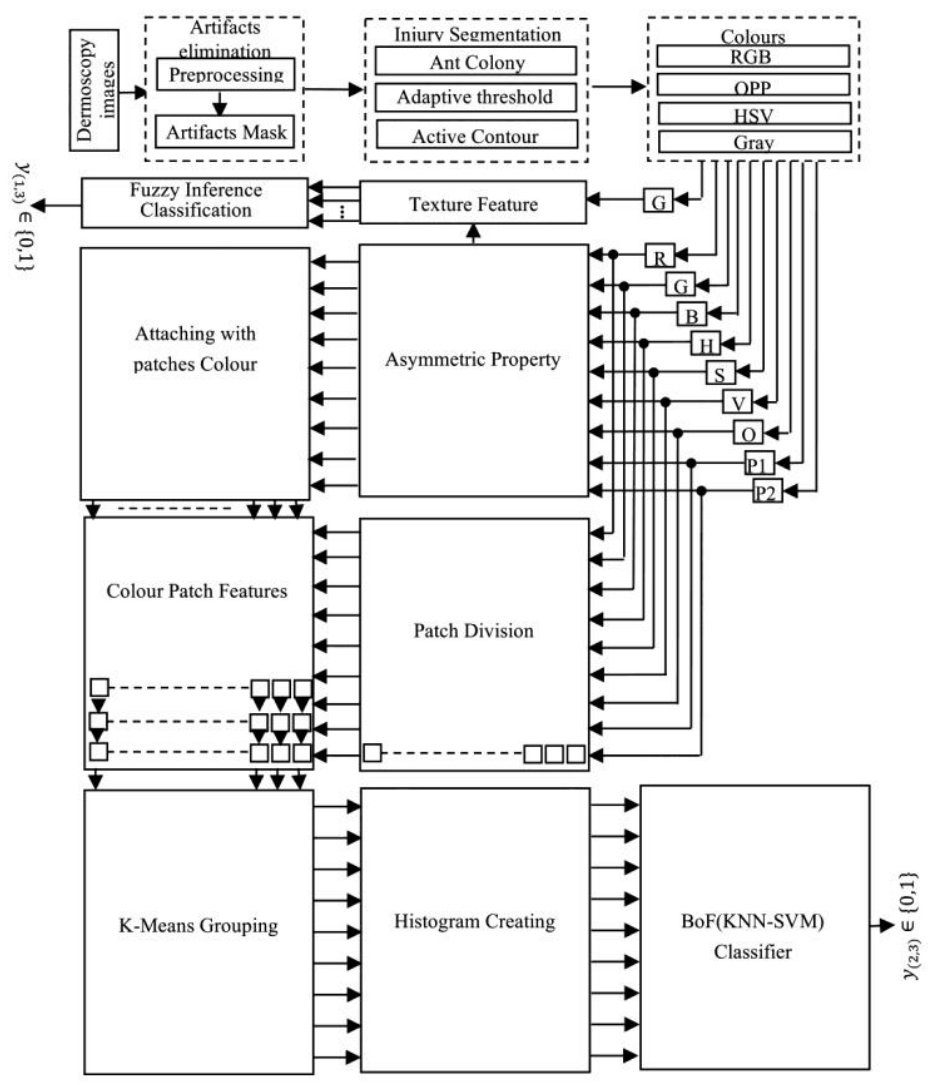

Figure 2. Block Diagram of Proposed skin lesion diagnostic System. 
Figure 2 shows the three stages of the proposed system: removing an artifact, a new contribution of segmentation process, and the main contributions of classification strategies. It demonstrates the examination of different colors spaces, which are tested to know the most important one, as well as the two Fuzzy and BoF classification systems, as well as the new contribution that led to the largest classification by adding asymmetry characteristic of tumor to each patch in the bag of words system.

\section{ARTIFACT REMOVAL}

Dermoscopic images often include the presence of artifacts such as illumination, dermoscopic gel, ink markings, vignette layers, ruler's marks, skin lines, veins, and hairs that could adversely affect the segmentation performance. Preprocessing was done to ensure that images have a consistent contrast between the part of the interest and neighboring areas by enhancing contrast. Secondly, methods to remove all artifacts (hairs, vignette layers, ink shuffles, rulers sign, skin lines, veins and ruler markings, etc.) must be used, by generating a binary mask that includes these artifacts only. First, the original image [Figure 3,(a)] is resized to a square fixed scale. An applied winner filter with a canny adaptive filter on the red channel of the RBG images for noise removing and edge is detected. The morphological opening operator was performed by lines structuring elements oriented in a different direction to obtain a binary mask, and artifact repair by image inpainting technique as illustrated in Figure 3(b). The database is provided as an international skin images collection in the 2019 ISBI challenge (ISIC,2019), and the ISBI web consists of dermoscopic frames with an expert clinician's floor truth segmentation mask.
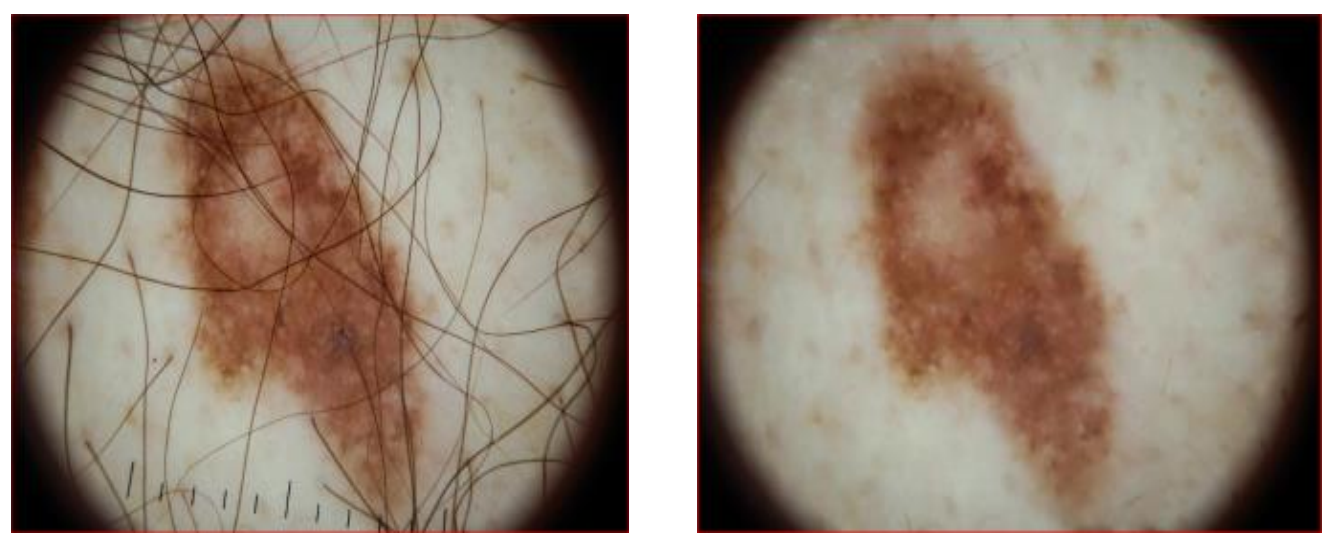

Figure 3. a) Dermoscopic lesion Picture. b) Artifact Removing Picture.

\section{SEGMENTATION STRATEGIES}

In order to take out the cancerous region of the healthy skin, the integration of three techniques to classify skin lesion was suggested. Triple-A segmentation strategies rely on the histogram distribution proposed. Decision Ant Colony Optimization (ACO) was used when entry image automatically classifies U-shape distribution, Active Contours Models (ACM) for bell-distribution, and Adaptive Threshold Technique (ATT) for J, reverse J shapes as shown in Figure 4. The proposed AAA strategies are improving segmentation accuracy compared with using any one of them alone. 


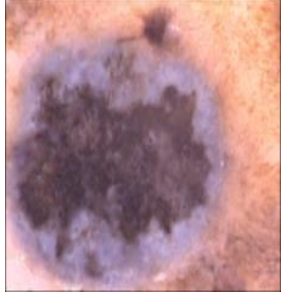

(a)

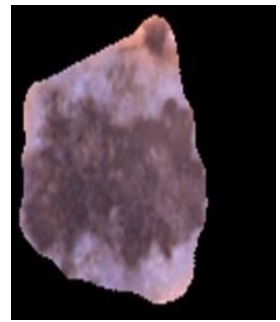

(d)ACO

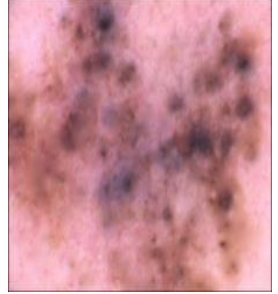

(b)

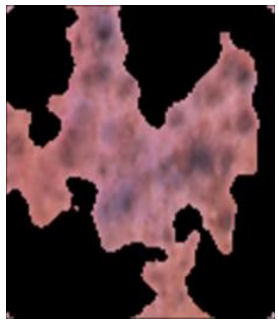

(e) $\mathrm{ACM}$

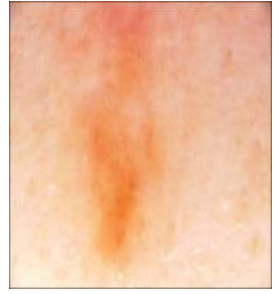

(c)

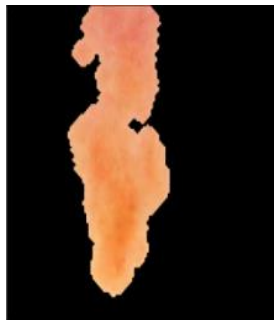

(f)ATT

Figure 4. (a,b,c)Original dermoscopic images (e,d,f)Segmented images

\section{CLASSIFICATION STRATEGIES}

Four recognition systems for melanoma detection were presented in this paper with a comparison between them. Firstly, describe the dermoscopic pictures by a set of global characteristics using the fuzzy classifier. Secondly, the dermoscopic picture is characterized by a BoF process depicted in Barata et al. (2014; 2013), and a set of key points was selected inside the specific regions. Then, we described each key by a vector of local characteristics to symbolize three color properties in a local patch centered at the key point. Local characteristics of each dermoscopy picture are allocated to the closest visual word and calculated in the histogram. A statistical classifier was taught to discriminate cancer by using the histogram of the visual words as an input. The third idea is adding an injury shape property (asymmetric or symmetric) with two previous systems, used as globally in the first one and appended to all local patches features of a BoF classifier to ensure which is the better one.

This paper attempts to figure out which of these procedures performs best in melanoma identification. The appropriate response is questionable provided that a skin injury is homogeneous, and the global thinking will most likely be the best because it can depict the sore by global features (Barata et al., 2014). Furthermore, skin sores regularly have differential structures such as pigment arranges, vascular system, spots, and streaks, which are limited and show up in explicit areas, and the global ones may not represent these components, and local strategies were smarter to depict them (Masood, 2016). Therefore, this paper provides a test of these four frameworks with a change of their parameters to prize better classification.

\section{First Classification System}

Fuzzy logic is somewhat similar to the functioning of the human brain. As the human mind while making a decision or arriving at a conclusion, at first, it collects some relevant data, and then some partial facts are generated from that data (Mathur, 2018). Fuzzy rule plays an important role in complex and mysterious systems (Hamad Y. A. and Naeem M. B.,2019). So, the first classification is done by utilizing fuzzy approach. 
Selection features process plays an important role in any classification system, so the extraction of useful ones is a challenging task. Many works use texture analysis, and the most usually utilized texture dealings are gotten from the Gray Level Cooccurrence Matrix (GLCM) (Masood, 2016),(Sheha,2016), (Barata et al.,2014), (Barata et al.,2013), (Kumar and Kumanan,2018), (Sreena and Lijiya,2018), (Sagar,2016), (Barata et al.,2015), (Thompson and Jeyakumar,2018), (Baji,2018), (Ruela,2012). These features are mining based on GLCM, which are Correlation, Autocorrelation, Contrast, Bunch Shade, Bunch Prominence, Dissimilarity, Entropy, Homogeneity, Energy, Maximum likelihood, Sum average, Sum of squares Variance, Sum variance, Variance variation, Sum entropy, Difference entropy, Data extent of correlation 1 and correlation 2, Inverse distinction homogenous, Inverse distinction normalized, and converse difference moment normalized (Barata et al., 2015).

The first phase starts by converting RGB frame to gray frame and testing many sets of features to select the most dominant ones, from GLCM features of Contrast, Correlation, Bunch Prominence, Dissimilarity, Homogeneity, Difference entropy, Variance variation, Data extent of correlation 1 and correlation 2, Inverse distinction homogenous, Inverse dissimilarity normalized, and Inverse dissimilarity moment normalized. The wisest features were tested using Mamdani Fuzzy Inference System (FIS) by creating a membership and used If-then rules (Alawad et al., 2018).

In the training phase, the skin features variable is fuzzified by applying Gaussian membership

$$
\exp \left[-\frac{1}{2}\left(\frac{x-c}{s}\right)^{m}\left[\begin{array}{c}
\text { where } \mathrm{c}, \mathrm{s} \text { represents the mean, standard deviation and } \mathrm{m} \text { represents } \\
\text { fuzzification factor }
\end{array}\right],\right.
$$

to represent the linguistic terms in the rules. Similarly, the output variable is based on the Mamdani fuzzy, having status as healthy skin or cancer skin. In this phase, the combined many sets of the feature include wavelet invariant moments, SIFT, Color-SIFT, DCT, different color space, and texture features that are tested for choosing the most effective features, and twelve of them are adopted in this phase. In the testing phase, 400 images are decomposed with all features to classify them into melanomas and nevi. The evolution metrics are Sensitivity (Se. $=79.5 \%$ ), Specificity $($ Sp. $=85 \%)$, and accuracy (Acc. $=82.5 \%)$.

\section{Second Classification System}

On the other hand, the use of a visual Bag of Words (BoW) can lead to a more accurate classification (Qi et al., 2019). Visual BoW extracts key point features, collects visual vocabulary, then gets a bag of visual words, and utilizes visual word vector to represent an image (Arun et al., 2019).

In clinical images, classification color features were investigated to disintegrate malignant melanoma lesions in most related research (Cardoso, 2019), (Barata, 2017). A skin picture description with local characteristics has been effectively utilized in many complex troubles, such as view acknowledgment and object recognition (Barata et al., 2014). Since difficult objects cannot be fashioned by a global form, so the BoF scheme was used to depict them by a set of local elements referred to as patches. A collection of key points was defined in the patch of size $\Delta \times \Delta$, and then we described each key point by a function vector, which represents data conveyed in the $\Delta \times \Delta$ patch size, which is centered at a key point. Patches are rejected if half or greater amount of its zone situate out the sore.

The training phase used a collection of labeled dermoscopic images to define BoF classifier parameters mentioned in Barata et al. (2014). At first, they are using a clustering algorithm to approximate the characteristic vectors in $\mathrm{F}$ by a centroids prototypes $(\mathrm{c} 1, \ldots, \mathrm{ck})$. All feature vectors in the teaching set are arranged to the closest model. A histogram that checks the event of every one of the models was calculated for all training 
images Ik. Each preparation image was portrayed by a visual words histogram $\mathrm{h}(\mathrm{k})$ with $\mathrm{K}$ bins. Due to the images classified by an expert in the training set, the classifier learns to predict their labels (melanoma or non-melanoma for the specified visual word histogram.

In a testing task, Compute the key points inside the injury of a new input image of its local characteristics. Local characteristics were then categorized utilizing the visual words dictionary, and the visual word histogram was constructed. Finally, the histogram was categorized using the learned classifier. Three color spaces, that is, RGB, HSV, and OPP, were tested to evaluate the most dominate set. Classifier execution for each color function was evaluated using various parameter combinations, changing the amount of histogram bins (nbins $\in\{25,50,128,256\}$ ), taking $\in\{16 \times 16,32 \times 32\}$ patch size and grouping the visual characteristics into $\in(10,4)$ clusters based on two classification techniques (kNN, SVM). Two separation functions (Euclidean, Cityblock) are calculated between features for kNN classifier. The kernel sort of the SVM is 'rbf' Gaussian Radial Basis Function with a sigma, with 1 default scaling factor. The best outcomes were gotten utilizing the opponent color space, $\mathrm{kNN}$ classifier, and distance form Euclidean in as abridged in Table 1.

Table 1. Three Colour Bag-of-Feature Results.

\begin{tabular}{|c|c|c|c|c|c|c|c|c|c|c|c|c|c|c|c|c|c|c|c|c|c|c|c|c|c|c|c|c|c|c|}
\hline \multicolumn{12}{|c|}{ RGB Local Features } & \multicolumn{9}{|c|}{ HSV Local Features } & \multicolumn{10}{|c|}{ OPP Local Features } \\
\hline \multirow{3}{*}{ 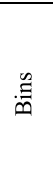 } & \multirow{3}{*}{ 营 } & \multirow{3}{*}{$\frac{\bar{s}}{\stackrel{5}{\Xi}}$} & \multicolumn{6}{|c|}{ KNN Classifier } & \multirow{2}{*}{\multicolumn{3}{|c|}{$\begin{array}{c}\text { SVM } \\
\text { Classifier }\end{array}$}} & \multicolumn{6}{|c|}{ KNN Classifier } & \multirow{2}{*}{\multicolumn{3}{|c|}{$\underset{\text { Classifier }}{\text { SVM }}$}} & \multicolumn{7}{|c|}{ KNN Classifier } & \multirow{2}{*}{\multicolumn{3}{|c|}{$\underset{\text { Classifier }}{\text { SVM }}$}} \\
\hline & & & \multicolumn{3}{|c|}{ Euclidean } & \multicolumn{3}{|c|}{ Cityblock } & & & & \multicolumn{3}{|c|}{ Euclidean } & \multicolumn{3}{|c|}{ Cityblock } & & & & \multicolumn{3}{|c|}{ Euclidean } & \multicolumn{4}{|c|}{ Cityblock } & & & \\
\hline & & & $\begin{array}{c}\text { Se. } \\
\%\end{array}$ & $\begin{array}{c}S p . \\
\%\end{array}$ & $\underset{\%}{A c .}$ & $\begin{array}{c}S e . \\
\%\end{array}$ & $\begin{array}{c}S p . \\
\%\end{array}$ & $\begin{array}{l}\text { Ac. } \\
\%\end{array}$ & $\begin{array}{c}\text { Se. } \\
\%\end{array}$ & $\begin{array}{c}S p . \\
\%\end{array}$ & $\begin{array}{c}\text { Ac. } \\
\%\end{array}$ & $\begin{array}{c}\text { Se. } \\
\%\end{array}$ & $\begin{array}{c}S p . \\
\%\end{array}$ & $\begin{array}{c}\text { Ac. } \\
\%\end{array}$ & $\begin{array}{c}\text { Se. } \\
\%\end{array}$ & $\begin{array}{c}S p . \\
\%\end{array}$ & $\begin{array}{r}\text { Ac. } \\
\%\end{array}$ & $\begin{array}{c}\text { Se. } \\
\%\end{array}$ & $\begin{array}{c}S p . \\
\%\end{array}$ & $\begin{array}{c}\text { Ac. } \\
\%\end{array}$ & $\begin{array}{r}S e . \\
\%\end{array}$ & $\begin{array}{c}S p \\
\%\end{array}$ & $\begin{array}{r}A c \\
\%\end{array}$ & $\begin{array}{c}S c \\
\%\end{array}$ & & $\begin{array}{c}S p . \\
\%\end{array}$ & $\begin{array}{c}\text { Ac. } \\
\%\end{array}$ & $\begin{array}{r}\text { Se. } \\
\%\end{array}$ & $\begin{array}{c}S p . \\
\%\end{array}$ & $\begin{array}{c}\text { Ac. } \\
\%\end{array}$ \\
\hline 25 & 32 & 10 & 60 & 52.5 & 54 & 100 & 2.5 & 22 & 100 & 5 & 24 & 70 & 97.5 & 92 & 100 & 5 & 24 & 70 & 25 & 34 & 90 & 85 & 86 & 50 & & 62.5 & 60 & 70 & 27.5 & 36 \\
\hline 25 & 32 & 4 & 70 & 57.5 & 60 & 100 & 0 & 20 & 100 & 5 & 24 & 80 & 90 & 88 & 100 & 10 & 28 & 100 & 10 & 28.5 & 100 & 85 & 88 & 50 & & 60 & 58.5 & 0 & 100 & 80 \\
\hline 25 & 16 & 10 & 50 & 45 & 46 & 100 & 2.5 & 22 & 80 & 12.5 & 26 & 80 & 90 & 88 & 100 & 10 & 28 & 80 & 40 & 48 & 90 & 85 & 86 & 7 & & 50 & 54 & 40 & 72.5 & 66 \\
\hline 25 & 16 & 4 & 70 & 55 & 58 & 100 & 0 & 20 & 80 & 32.5 & 42 & 80 & 90 & 88 & 100 & 5 & 24 & 100 & 5 & 24 & 90 & 85 & 86 & 50 & & 37.5 & 40 & 20 & 72.5 & 62 \\
\hline 50 & 32 & 10 & 30 & 75 & 66 & 100 & 0 & 20 & 100 & 25 & 40 & 70 & 92.5 & 88 & 100 & 0 & 20 & 100 & 20 & 36 & 100 & 80 & 84 & 10 & & 12.5 & 30 & 30 & 40 & 38 \\
\hline 50 & 32 & 4 & 40 & 62.5 & 58 & 100 & 0 & 20 & 100 & 22.5 & 38 & 80 & 78.5 & 86 & 100 & 0 & 20 & 100 & 10 & 28 & 100 & 87. & 90 & 10 & & 12.5 & 30 & 20 & 72.5 & 62 \\
\hline 50 & 16 & 10 & 50 & 80 & 74 & 100 & 0 & 20 & 100 & 25 & 40 & 80 & 92.5 & 90 & 100 & 0 & 20 & 100 & 17.5 & 34 & 80 & 80 & 80 & 10 & & 12.5 & 30 & 10 & 57.5 & 48 \\
\hline 50 & 16 & 4 & 70 & 67.5 & 68 & 100 & 0 & 20 & 100 & 20 & 36 & 80 & 87.5 & 86 & 100 & 0 & 20 & 100 & 0 & 20 & 100 & 85 & 88 & 10 & & 12.5 & 30 & 20 & 80 & 68 \\
\hline 128 & 32 & 10 & 20 & 85 & 72 & 100 & 0 & 20 & 100 & 7.5 & 26 & 70 & 92.5 & 88 & 100 & 0 & 20 & 80 & 22.5 & 34 & 80 & 92. & 90 & 10 & & 0 & 20 & 80 & 32.5 & 42 \\
\hline 128 & 32 & 4 & 30 & 70 & 62 & 100 & 0 & 20 & 100 & 5 & 24 & 80 & 87.5 & 86 & 100 & 0 & 20 & 100 & 12.5 & 30 & 80 & 90 & 88 & 10 & & 0 & 20 & 20 & 90 & 76 \\
\hline 128 & 16 & 10 & 40 & 90 & 80 & 100 & 0 & 20 & 100 & 15 & 32 & 90 & 92.5 & 92 & 100 & 0 & 20 & 90 & 50 & 58 & 100 & 95 & 96 & 10 & & 0 & 20 & 60 & 62.5 & 62 \\
\hline 128 & 16 & 4 & 40 & 82.5 & 74 & 100 & 0 & 20 & 100 & 25 & 40 & 80 & 95 & 92 & 100 & 0 & 20 & 100 & 10 & 28 & 90 & 87. & 88 & 10 & & 0 & 20 & 70 & 95 & 90 \\
\hline 256 & 32 & 10 & 10 & 95 & 78 & 100 & 0 & 20 & 100 & 22.5 & 38 & 60 & 87.5 & 82 & 100 & 0 & 20 & 100 & 22.5 & 38 & 90 & 95 & 94 & 10 & & 0 & 20 & 40 & 45 & 44 \\
\hline 256 & 32 & 4 & 20 & 87.5 & 74 & 100 & 0 & 20 & 100 & 22.5 & 38 & 70 & 90 & 86 & 100 & 0 & 20 & 100 & 5 & 24 & 90 & 87. & 88 & 10 & & 0 & 20 & 20 & 75 & 64 \\
\hline 256 & 16 & 10 & 0 & 97.5 & 78 & 100 & 0 & 20 & 100 & 25 & 40 & 60 & 87.5 & 82 & 100 & 0 & 20 & 100 & 22.5 & 38 & 100 & 72. & 76 & 10 & & 0 & 20 & 20 & 57.5 & 50 \\
\hline 256 & 16 & 4 & 0 & 95 & 76 & 100 & 0 & 20 & 100 & 22.5 & 38 & 70 & 85 & 82 & 100 & 0 & 20 & 100 & 7.5 & 26 & 100 & 85 & 88 & 10 & & 0 & 20 & 20 & 85 & 72 \\
\hline
\end{tabular}

\section{Third Classification Procedure}

Consolidating various descriptors of a similar class may improve the outcomes. According to the clinical observation, asymmetry of the pattern was given the highest weight for diagnosis. To check the assumption of previous systems, asymmetric ones are mixed with public texture with and neighboring colors. The asymmetry lesion property was essential in the examination of the skin disease; it is the first of the ABCD strategy to decide a harmful 
melanoma. So, asymmetric global property was determined to enhance diagnosis by contrasting of injury region difference in form and colors. Then, we attached it to the first scheme with twelve texture options that utilized fuzzy reasoning classifier to check recent 13 properties and validate the advantage of adding asymmetric to the first scheme. And the whole asymmetric feature was distributed to the patches, features of coaching frames, and appending to its same patched color characteristics demonstrated in Figure 2.

Since the local extraction feature based on the BoF classifier relies upon various parameters, so each of them was varied to reap the high-quality classification rankings and employ this procedure by using $\{16 \times 16,32 \times 32\}$ patch sizes and ranging the quality of histogram nbins $\in\{25,50, . ., 256\}$. The visual terms are acquired by implementing a $\mathrm{k}$-means clustering for coaching vector features. The tried images were characterized as malignant melanoma or not by testing the SVM and kNN algorithm. Two distances (Euclidean, and Cityblock) have been regarded to achieve a better one. Table 2 verifies the experimental results achieved by appending asymmetric function and scattered with all patches based on BoF classifier. This feature role direction is to be considerably more essential rather than it is used as acquired in the complete lesion. RGB color characteristics accomplish healthier outcomes because of its exhibition with asymmetric function, resulting in $\mathrm{Se} .=100 \%$ and $\mathrm{Sp} .=97.5 \%$ utilizing $\mathrm{kNN}$ classifier and cityblock separation. The OPP asymmetric color delays the outcomes for the same factors (Se. $=100 \%$, Sp. $=95 \%$ ). Other than this, attaching asymmetric characteristics to the primary global blurry scheme ascended sensitivity and specificity to Se. $=84.6 \%$ and Sp. $=90 \%$, respectively.

Table 2. Result of Appending Asymmetric Feature with three Colour Bag-of-Features.

\begin{tabular}{|c|c|c|c|c|c|c|c|c|c|c|c|c|c|c|c|c|c|c|c|c|c|c|c|c|c|c|c|c|c|}
\hline \multirow{4}{*}{ 色 } & \multirow{4}{*}{ 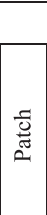 } & \multirow{4}{*}{ 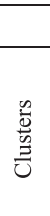 } & \multicolumn{9}{|c|}{ RGB Image Space } & \multicolumn{9}{|c|}{ HSV Image Space } & \multicolumn{9}{|c|}{ OPP Image Space } \\
\hline & & & \multicolumn{6}{|c|}{ KNN-Classifier } & \multirow{2}{*}{\multicolumn{3}{|c|}{$\underset{\text { Classifier }}{\text { SVM }}$}} & \multicolumn{6}{|c|}{ KNN-Classifier } & \multirow{2}{*}{\multicolumn{3}{|c|}{$\begin{array}{c}\text { SVM } \\
\text { Classifier }\end{array}$}} & \multicolumn{6}{|c|}{ KNN-Classifier } & \multirow{2}{*}{\multicolumn{3}{|c|}{$\begin{array}{l}\text { SVM- } \\
\text { Classifier }\end{array}$}} \\
\hline & & & \multicolumn{3}{|c|}{ Euclidean } & \multicolumn{3}{|c|}{ Cityblock } & & & & \multicolumn{3}{|c|}{ Euclidean } & \multicolumn{3}{|c|}{ Cityblock } & & & & \multicolumn{3}{|c|}{ Euclidean } & \multicolumn{3}{|c|}{ Cityblock } & & & \\
\hline & & & $\begin{array}{c}\text { Se. } \\
\%\end{array}$ & $\begin{array}{c}\text { Sp. } \\
\%\end{array}$ & $\begin{array}{c}\mathrm{Ac} \\
\%\end{array}$ & \begin{tabular}{c|}
$\mathrm{Se}$ \\
$\%$
\end{tabular} & $\begin{array}{l}\text { Sp. } \\
\%\end{array}$ & $\begin{array}{l}\text { Ac. } \\
\%\end{array}$ & $\begin{array}{c}\mathrm{Se} \\
\%\end{array}$ & $\begin{array}{c}\text { Sp. } \\
\%\end{array}$ & $\begin{array}{c}\mathrm{Ac} \\
\%\end{array}$ & $\begin{array}{l}\text { Se. } \\
\%\end{array}$ & $\begin{array}{c}\text { Sp. } \\
\%\end{array}$ & $\begin{array}{c}\text { Ac. } \\
\%\end{array}$ & \begin{tabular}{c|} 
Se. \\
$\%$
\end{tabular} & $\begin{array}{c}\text { Sp. } \\
\%\end{array}$ & $\begin{array}{c}\text { Ac. } \\
\%\end{array}$ & \begin{tabular}{|c|} 
Se. \\
$\%$
\end{tabular} & $\begin{array}{c}\text { Sp. } \\
\%\end{array}$ & $\begin{array}{c}\text { Ac. } \\
\%\end{array}$ & \begin{tabular}{|c|} 
Se. \\
$\%$
\end{tabular} & $\begin{array}{c}\text { Sp. } \\
\%\end{array}$ & $\begin{array}{c}\text { Ac. } \\
\%\end{array}$ & $\begin{array}{c}\text { Se. } \\
\%\end{array}$ & $\begin{array}{l}\text { Sp. } \\
\%\end{array}$ & $\begin{array}{c}\text { Ac. } \\
\%\end{array}$ & $\begin{array}{c}\text { Se. } \\
\%\end{array}$ & $\begin{array}{c}\text { Sp. } \\
\%\end{array}$ & $\begin{array}{c}\text { Ac. } \\
\%\end{array}$ \\
\hline 25 & 32 & 10 & 100 & 57.5 & 66 & 90 & 7.5 & 88 & 90 & 92.5 & 92 & 100 & 82.5 & 86 & 80 & 92.5 & 90 & 100 & 87.5 & 90 & 100 & 87.5 & 90 & 50 & 100 & 90 & 50 & 100 & 90 \\
\hline 25 & 32 & 4 & 0 & 50 & 60 & 100 & 72.5 & 78 & 90 & 87.5 & 88 & 100 & 87.5 & 90 & 100 & 90 & 92 & 80 & 100 & 96 & 100 & 82.5 & 86 & 30 & 100 & 86 & 0 & 100 & 80 \\
\hline 25 & 16 & 10 & 0 & 57.5 & 66 & 80 & 90 & 88 & 90 & 92.5 & 92 & 100 & 87.5 & 90 & 80 & 92.5 & 90 & 100 & 87.5 & 90 & 100 & 82.5 & 86 & 0 & 100 & 88 & 20 & 100 & 84 \\
\hline 25 & 16 & 4 & 100 & 52.5 & 62 & 90 & 87.5 & 88 & 90 & 92.5 & 92 & 100 & 90 & 92 & 100 & 87.5 & 90 & 100 & 82.5 & 86 & 100 & 80 & 84 & 60 & 100 & 92 & 10 & 100 & 82 \\
\hline 50 & 3 & 10 & 100 & 82.5 & 86 & 80 & 92.5 & 90 & 90 & 92.5 & 92 & 0 & 87.5 & 90 & 80 & 92.5 & 0 & 80 & 92.5 & 90 & 0 & 87.5 & 0 & 100 & 2.5 & 86 & 80 & 100 & 96 \\
\hline 50 & 3 & 4 & 0 & 60 & 68 & 100 & 2.5 & 78 & 90 & 87.5 & 88 & 00 & 90 & 92 & 100 & 72.5 & 78 & 90 & 92.5 & 92 & 100 & 7.5 & 90 & 100 & 85 & 88 & 40 & 100 & 88 \\
\hline 50 & 1 & 10 & a & 82.5 & 86 & 80 & 5 & 9 & 90 & 92.5 & 92 & 0 & 87.5 & 90 & 80 & 92.5 & 90 & 801 & 100 & 96 & 100 & 87.5 & 90 & 100 & 82.5 & 86 & 40 & 100 & 88 \\
\hline 50 & 16 & 4 & 100 & 57.5 & 66 & 90 & 7.5 & 88 & 90 & 92.5 & 92 & 100 & 87.5 & 90 & 100 & 90 & 92 & 80 & 92.5 & 90 & 100 & 80 & 84 & 100 & 85 & 88 & 20 & 100 & 84 \\
\hline 128 & 32 & 10 & & 82.5 & 8 & & & 90 & 90 & & 9 & 0 & 5 & 86 & 0 & 72.5 & 78 & 80 & 0 & 6 & 0 & 87.5 & 0 & 0 & 7.5 & 90 & 70 & 100 & 94 \\
\hline 128 & 32 & 4 & 100 & 72.5 & 78 & 100 & 85 & 88 & 90 & 92.5 & 92 & 100 & 90 & 92 & 80 & 92.5 & 90 & 100 & 82.5 & 86 & 100 & 80 & 4 & 100 & 87.5 & 90 & 30 & 100 & 86 \\
\hline 128 & 1 & 1 & & 82.5 & 5 & 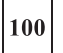 & .5 & 9 & 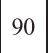 & 5 & 8 & 0 & 5 & 90 & 0 & 5 & 94 & 80 & 5 & 0 & 0 & 2.5 & 6 & 0 & 08 & 96 & 30 & 100 & 36 \\
\hline 128 & 10 & 4 & 100 & 85 & 80 & 80 & 92.5 & 90 & 100 & 72.5 & 78 & 100 & 82.5 & 86 & 80 & 92.5 & 90 & 90 & 100 & 98 & 100 & 92.5 & 94 & 100 & 87.5 & 90 & 10 & 100 & 82 \\
\hline 256 & 32 & 10 & 100 & 72.5 & 78 & 80 & 9 & 88 & 90 & 92.5 & 92 & 100 & 90 & 92 & 100 & 72.5 & 78 & 80 & 92.5 & 90 & 100 & 87.5 & 0 & 100 & 85 & 88 & 60 & 100 & 92 \\
\hline 256 & 32 & 4 & 100 & 72.5 & 78 & 80 & 92.3 & 90 & 90 & 92.5 & 92 & 100 & 90 & 92 & 100 & 87.5 & 90 & 100 & 82.5 & 86 & 100 & 03 & 88 & 100 & 87.5 & 90 & 40 & 100 & 88 \\
\hline 256 & 16 & 10 & 0 & 82.5 & 86 & 90 & 8 & 88 & 100 & 72.5 & 78 & 100 & 87.5 & 90 & 100 & 90 & 92 & 80 & 100 & 96 & 100 & 92.5 & 4 & 100 & 2.5 & 86 & 40 & 100 & 88 \\
\hline 256 & 16 & 4 & 100 & 72.5 & 78 & 100 & 72.5 & 78 & 90 & 87.5 & 88 & 100 & 82.5 & 86 & 100 & 72.5 & 78 & 100 & 82.5 & 86 & 100 & 82.5 & 86 & 100 & 87.5 & 90 & 40 & 100 & 88 \\
\hline
\end{tabular}




\section{COMPARISON RESULTS}

To compare the benefits of the proposed thinks, figure 5 was plotted between accuracies and consuming timeof two better systems, utilizing three color spaces only and asymmetric patch attaching idea. Other than, tables 3 clears the performance of four presented systems and averment the local BoF accomplishing preferable outcomes over the global scheme utilizing FIS systems. Computing local characteristics with asymmetric ones needs extra time compared with other ones, as in the container of the local scheme.

\section{DISCUSSION}

The classification was utilized within three main ideas: (1) describing skin images by global parameters blurry classifier, (2) histogram of local colour using a BoF, and (3) appending asymmetry of lesion with greatest clinical feature with the two preceding ideas. The BoF second classifier gives greater overall performance than the global first system. Their performance was calculated using RBG, HSV, and OPP colour spaces, 25-256 bins, 10, 4 clusters with two patch sizes $(16 \times 16,32 \times 32)$ due to estimate optimal one regarding $\mathrm{kNN}$.

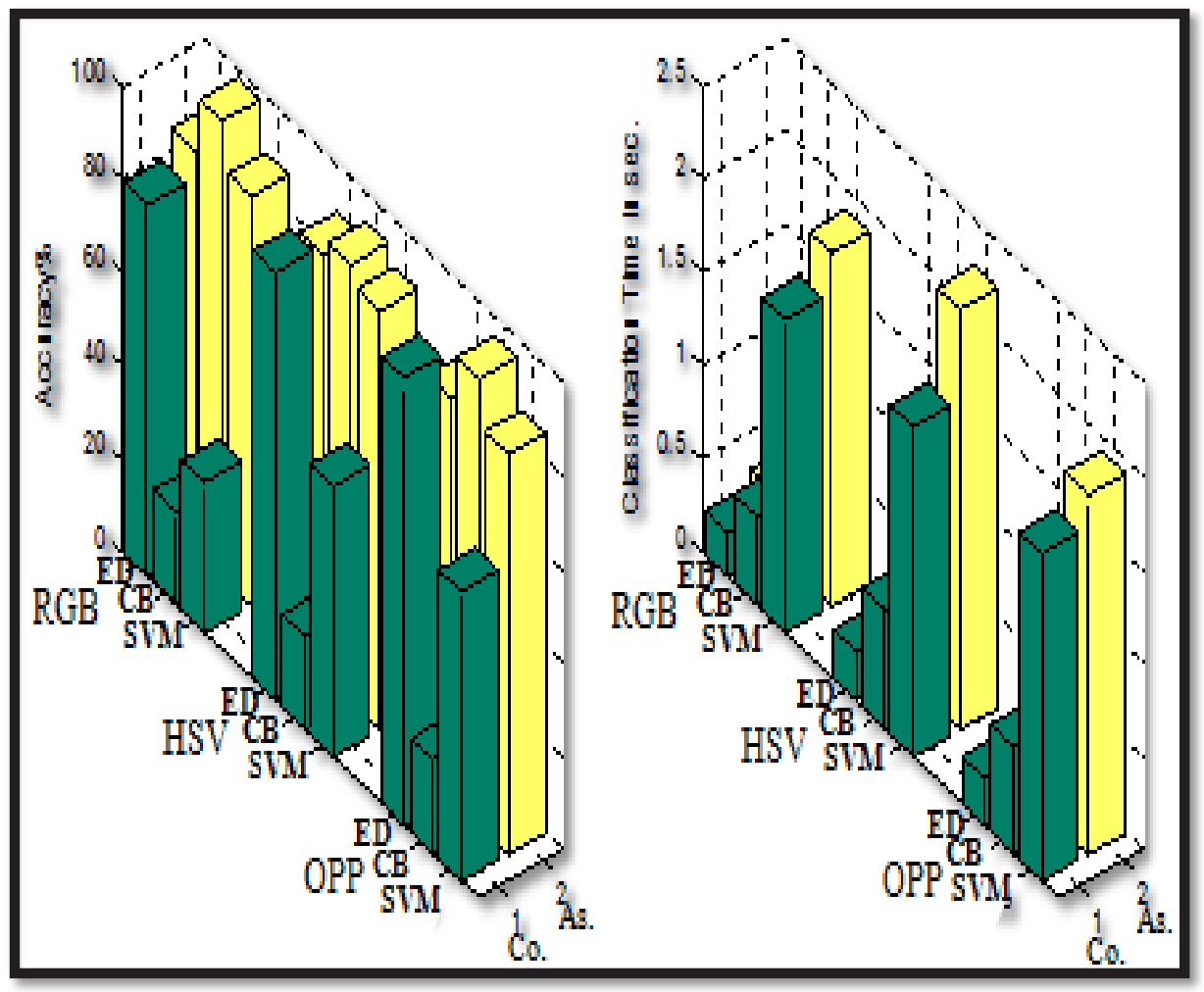

Figure 5. Accuracy and classification time of the best case (16×16 Patch size, 128 - bins $\& 10$-cluster) colour only(Co) and Colour with Asymmetric(As). 
Table 3. Comparison Results of Four Systems.

\begin{tabular}{|c|c|c|c|c|c|}
\hline \multirow{2}{*}{ System } & \multirow{2}{*}{ Feature } & \multirow{2}{*}{ Classifier } & \multirow{2}{*}{ Distance } & \multicolumn{2}{|c|}{ Time(s) } \\
\hline & & & & Feature & Classify \\
\hline First & Texture & FIS & & 2.5034 & 0.2425 \\
\hline \multirow{9}{*}{ Second } & \multirow{3}{*}{ RGB } & \multirow{2}{*}{$\mathrm{kNN}$} & Euclidean & \multirow{3}{*}{1.4205} & 0.2413 \\
\hline & & & Cityblock & & 0.4777 \\
\hline & & SVM & & & 1.6756 \\
\hline & \multirow{3}{*}{ HSV } & \multirow{2}{*}{$\mathrm{kNN}$} & Euclidean & \multirow{3}{*}{2.2941} & 0.2715 \\
\hline & & & Cityblock & & 0.6368 \\
\hline & & SVM & & & 1.7673 \\
\hline & \multirow{3}{*}{ OPP } & \multirow{2}{*}{$\mathrm{kNN}$} & Euclidean & \multirow{3}{*}{1.5293} & 0.2547 \\
\hline & & & Cityblock & & 0.5771 \\
\hline & & SVM & & & 1.7514 \\
\hline Third & $\begin{array}{c}\text { Texture \& } \\
\text { Asym. }\end{array}$ & FIS & & 3.1447 & 0.2682 \\
\hline \multirow{9}{*}{ Fourth } & \multirow{3}{*}{ RGB \& Asym. } & $1-$ & Euclidean & \multirow{3}{*}{1.7646} & 0.3107 \\
\hline & & & Cityblock & & 0.7194 \\
\hline & & SVM & & & 1.8884 \\
\hline & \multirow{3}{*}{ HSV \& Asym. } & $\mathrm{kNN}$ & Euclidean & \multirow{3}{*}{2.4034} & 0.3492 \\
\hline & & & Cityblock & & 0.7379 \\
\hline & & SVM & & & 2.2566 \\
\hline & \multirow{3}{*}{ OPP \& Asym. } & 1) & Euclidean & \multirow{3}{*}{2.0374} & 0.3457 \\
\hline & & Riviv & Cityblock & & 0.648 \\
\hline & & SVM & & & 1.9279 \\
\hline
\end{tabular}

Classifier based on CB and ED distance metrics is used to compute the likeness of feature vectors. The OPP Color space representation that gives the best outcomes ( $\mathrm{Se} .=100 \%$ and $\mathrm{Sp} .=95 \%$ ) is the adversary by taking 128 , 10 , and $16 \times 16$ parameters. $\mathrm{kNN}$ works higher performance than SVM and ED acquiring better results than $\mathrm{CB}$ aloofness.

Appending asymmetric with a global GLCM feature in first blurry classifier also rose its performance. Moreover, the global asymmetric feature was extracted and distributed to all patches color BoF features models, which performs higher as a system result, and RGB offers better testing space due to its goodness of asymmetric description. The overall evaluation metrics are (Se. $=100 \%, \mathrm{Sp} .=97.6 \%$ and an $\mathrm{Acc} .=98.26 \%$ ) obtained by $\mathrm{kNN}$ classifier utilizing $\mathrm{CB}$ distance. So, the appending strategies are a vast promise in on the increase a quantitative of classifying skin injury. 


\section{CONCLUSION}

The first contribution of this paper is to use three strategies: ant colony optimization, active contour models, and adaptive threshold technique for injury segmentation. The proposed AAA process raised the segmentation accuracy to $96.87 \%$. A second contribution is to use global and local feature together in classification stage. Thirdly, many different features, colors, distances, classifiers, etc. have been tested to identify the most accurate as demonstrated in tables 1 and 2. The proposed procedure can be done with short time verified in table 3 and simple resources compared with deep learning algorithms. Future Deep learning research can be done utilizing the best classifier with its special parameters.

\section{ACKNOWLEDGMENT}

The authors would like to express their deep gratitude and thanks to the International Skin Imaging Agency 2019 (ISIC2019) for publishing a wide range of skin images adopted as a reference for many researches.

\section{REFERENCES}

Adjed F. (2017), "Skin Cancer segmentation and Detection Using Total Variation and Multiresolution Analysis", Ph.D thesis, Thèse de doctorat de Universiti Teknologi PETRONAS et l'Université Paris-Saclay préparée à l'universiti Teknologi PETRONAS et l'université d'Evry Val d'Essonne.

Alawad A. M., Abdul Rahman F. D., Khalifa O. O., Abdul Malek N.(2018), "Fuzzy Logic based Edge Detection Method for Image Processing", International Journal of Electrical and Computer Engineering (IJECE) Vol. 8, No. 3, Pp. 1863 1869.

Arun K. S. , Govindan V. K. , Kumar S. D.(2019), "Enhanced bag of visual words representations for content based image retrieval: a comparative study", Artificial Intelligence Review, https://doi.org/10.1007/s10462019-09715-6.

Baji F. S. (2018), "Features Extraction Techniques for Medical Images Diagnosis and Retrieval", Ph.D thesis, Doctor of Philosophy in Computer Science and Information Technology, University of CRAIOVA, Romania.

Barata C. (2017), "Automatic Detection of Melanomas Using Dermoscopy Images", Ph.D. thesis, Universidade De LISBOA Instituto superior Tecnico, Electrical and Computer Engineering.

Barata C., Celebi M. E., and Marques J. S. (2015), "A Survey of Feature Extraction in Dermoscopy Image Analysis of Skin Cancer", Journal of LATEX , vol. 14, no. 8, p.1-14.

Barata C., Ruela M., Francisco M., Mendonça T., and Marques J. S. (2013), “Two systems for the detection of melanomas in dermoscopy images using texture and Colour features", IEEE Systems Journal.

Barata C., Ruela M., Mendonça T. and Marques J. S. (2014), “A bag-of-features approach for the classification of melanomas in dermoscopy images: the role of Colour and texture descriptors", Computer Vision Techniques for the Diagnosis of Skin Cancer, Series in BioEngineering, DOI:10.1007/978-3-642-39608-3_3, C) Springer-Verlag Berlin Heidelberg.

Cardoso T. M. (2019), "Skin Cancer Detection Using Sparse Coding", MSc. Thesis in Electrical and Computing Engineering, Tècnico Lisboa. 
Garbe C., Peris K., Hauschild A., Saiag P., Middleton M., Bastholt L., Grob J., Malvehy J., Newton-Bishop J., Stratigos A., Pehamberger H. and Eggermont A. (2016), "Diagnosis and treatment of melanoma. European consensus-based interdisciplinary guideline Update 2016", Elseiver European Journal of Cancer(EJC) 63 p.201-217.

Hamad Y. A. and Naeem M. B.(2019), "Detection of Brain Tumor in MRI Images, Using a Combination of Fuzzy C-Means and Thresholding", International Journal of Advanced Pervasive and Ubiquitous Computing Volume 11, Issue 1, Pp.45-60.

ISIC (2019) skin Lesion Analysis Towards Melanoma Detection, https://challenge2019.isic-archive.com.

Kawahara J., Daneshvar S. , Argenziano G., and Hamarneh G. (2018), "7-Point Checklist

and Skin Lesion Classification using Multi-Task Multi-Modal Neural Nets", JBHI-Special issue on Skin Lesion Image Analysis for Melanoma Detection, IEEE, Pp. 1-8.

Kumar S. M. and Kumanan T. (2018), "Analysis on Skin Lesion Classification Systems and

Dermoscopic Feature Analysis for Melanoma", International Journal for Research in Applied Science \& Engineering Technology (IJRASET), Vol. 6 Issue III, Pp.1971-1978.

Lone I. A. and Kaur M. (2020), "Improved Convolutional Neural Network Based Segmentation and Detection of Skin Cancer from Dermoscopy Images using MSER Descriptor", International Journal of Engineering Sciences \& Research Technology, 9(2), Pp.80-90.

Machado M., Pereira J. and Fonseca-Pinto R. (2016), "Reticular pattern detection in dermoscopy: an approach using Curvelet Transform", Research on Biomedical Engineering, DOI: http://dx.doi.org/10.1590/24464740.00315 .

Madooei A., Drew M. S. and Hajimirsadeghi H. (2018), "Learning to Detect Blue-white Structures in Dermoscopy Images with Weak Supervision", http://www.iee.org/publications_standards/publications /rights, IEEE Journal of Biomedical and Health Informatics, DOI 10.1109/JBHI.

Masood A. (2016), "Developing Improved Algorithms for Detection and Analysis of Skin Cancer", Ph.D thesis, Faculty of Engineering and Information Technology University of Technology, Sydney.

Mathur N., Meena Y. K., Mathur S. and Mathur D.(2018), "Detection of Brain Tumor in MRI Image through Fuzzy Based Approach”, DOI: 10.5772/intechopen.71485, Pp.47-62.

Qi Y., *, Zhanga G. , and Li Y.(2019), "Image Classification Model Using Visual Bag of Semantic Words", Pattern Recognition and Image Analysis, 2019, Vol. 29, No. 3, pp. 404-414.

Ruela A. M. (2012), "What is the role of Colour in dermoscopy analysis?", M.Sc. Thesis in Biomedical Engineering, Tècnico Lisboa.

Sagar C. (2016), "Detection of Melanoma from Consumer Camera Imagesof Skin Lesion Using Image Processing", MS.c Dissertation, Technology in Biomedical Engineering, School of Biomedical Engineering National institute of technology.

Sao P., Choudhary B. and Pandey V. (2018), "The Diagnosis and Analysis on the Skin Cancer Detection Using Pattern Recognition Technology", ICONIC Research and Engineering Journals(IRE),| vol. 2 Issue 6, p.81-86.

Sheha M. A., Sharwy A. and Mabrouk M. S. (2016), "Automated Imaging System for Pigmented Skin Lesion Diagnosis", (IJACSA) International Journal of Advanced Computer Science and Applications, vol. 7, no. 10, Pp. 242-254. 
Sreena S. and Lijiya A. (2018), "Survey on Skin Lesion Analysis towards Melanoma Detection", JCSE International Journal of Computer Sciences and Engineering, vol.-6, Issue-5.

Tan T. Y. (2019), "Intelligent Skin Cancer Detection using Enhanced Particle Swarm Optimization", Ph.D thesis, in the Faculty of Computer and Information Sciences, University of Northumbria at Newcastle.

Thompson F. and Jeyakumar M. K (2018), "Pattern Classification of Melanoma by Local Features Using BoF Based Spatial Encoding", International Journal of Engineering and Technology (IJET), Vol 9, No 6, Pp. 45274534.

Xie F., Fan H., Li Y., Jiang Z., Meng R., and Bovik A. (2017), "Melanoma Classification on Dermoscopy Images Using a Neural Network Ensemble Model", IEEE Transactions on medical Imaging, Vol. 36, NO. 3, Pp. 849-858. 